\title{
Lateral Forehead Flap in Neglected Giant Basal Cell Carcinoma of the Nose: A Case Report
}

\author{
Jonathan Kevin, Renate Parlene Marsaulina, Alberta Jesslyn Gunardi, Irena Sakura Rini
}

Department of Plastic, Reconstructive, and Aesthetic Surgery, Dharmais Cancer Hospital, Jakarta, Indonesia

\section{ARTICLE INFO}

Received : 19 July 2019

Reviewed : 15 August 2019

Accepted : 20 November 2019

\section{Keywords:}

lateral forehead flap, neglected giant basal cell carcinoma, large facial defect, nasal reconstruction

\author{
*Corresponding author: \\ Jonathan Kevin \\ Department of Plastic, Reconstructive \\ and Aesthetic Surgery, Dharmais \\ Cancer Hospital, Jakarta, Indonesia \\ johnkevinleon@yahoo.co.id
}

\author{
ABSTRACT
}

Introduction: Neglected basal cell carcinoma (BCC) of the nose can grow into giant BCCs, rare cases with extensive nasal defects. Such large defects would require complex reconstruction such as free flaps or multiple local flaps. Lateral forehead flap may provide a simpler alternative with good functional and cosmetic results.

Case Presentation: We present a case of a 76-year-old man with neglected giant BCC of nose extending to right lower eyelid and upper lip. Wide excision of the tumor leaves a $12 \mathrm{~cm} \times 10 \mathrm{~cm}$ defect. Reconstruction was performed using lateral forehead flap and donor site was covered with split-thickness skin graft from thigh. The second surgery was done after four months to create nostrils and wider eye-opening. Six months later, flap was viable and there was no sign of recurrence. Nasal reconstruction is planned to further improve cosmetics. In this case, neglect is due to low social-economic status and adaptation to painless tumors. The use of lateral forehead flap allows for simpler and faster surgery suitable for elderly. Delayed reconstruction was needed to ensure optimal tissue healing.

Conclusions: Neglected BCC causes disfigurement with remarkable morbidity, requiring complex reconstruction. The lateral forehead flap is a simple and reliable reconstruction method for extensive nasal defects with good functional and cosmetic outcomes.

\section{INTRODUCTION}

Basal cell carcinoma (BCC) is the most common malignancy worldwide, especially in fair-skinned people. Along with squamous cell carcinoma (SCC), these malignancies are grouped as non-melanoma skin cancer (NMSC) with an incidence of 18-20 times higher than melanoma [1]. American Cancer Society reported an estimation of 5.4 million NMSC cases are diagnosed each year, comprising of $80 \%$ BCC and $20 \%$ SCC [2]. The worldwide incidence rate of BCCs has been increasing $3-10 \%$ per year in the last decades and predicted to keep rising due to higher life expectancy and ultraviolet radiation exposure. Nevertheless, the epidemiology of BCC is understudied as most large cancer registries exclude NMSC from their records [3].

The nature of BCC is slow-growing with the low metastasis and mortality rate. However, BCC is associated with remarkable morbidity when treatment is delayed. $\mathrm{BCC}$ progression can invade surrounding tissues, causing local destruction and disfigurement. About $85-90 \%$ cases of BCC occur on the head, greatly affecting facial appearance of patients [4]. When neglected, BCC can grow larger than $5 \mathrm{~cm}$ in diameter and develop into giant BCC (GBCC). GBCCs are rare cases, comprising
$0.5 \%$ of all $\mathrm{BCC}$ cases. It is more aggressive and capable of invading underlying deep tissues or extradermal structures with infiltration to the dermis [5]. Although visible and easily identified by health professionals, neglected BCC cases may still be discovered due to patients being afraid of diagnoses and treatments or becoming accustomed to the slow-growing and painless tumor. Other factors affecting patient unawareness towards the danger of delayed diagnoses include old age, low social-economic status, and unhygienic habit [6].

Surgery is still the main treatment for GBCCs. Removal of large tumors on the face with proper margin while retaining functionality and aesthetics can be challenging. Moreover, it requires complex reconstruction techniques such as free flaps or multiple local flaps. Lateral forehead flap (LFF) or previously called temporal flap has been introduced decades ago for facial and intraoral defects [7]. LFFs utilize a wide forehead area to close large facial defects from GBCCs. It truly replaces like-with-like for its similar color and hairless surface with the facial or nasal skin. It is also well vascularized by temporal arteries, providing immediate covering skin for vital structures or raw surfaces. LFF as a local flap provides a simpler alternative for extensive nasal defects with good functional and cosmetic results [8]. 


\section{CASE PRESENTATION}

A 76 year old man was admitted to the Department of Plastic Surgery, Dharmais Cancer Hospital, due to large ulceration on his nose. It started as a small mole on the right side of the nose that bled after scratch 4 years ago. The wound was treated with povidone-iodine solution but only temporarily healed and easily bled again. The patient was taken to the hospital for biopsy, diagnosed with basal cell carcinoma and advised for chemotherapy. He declined due to the economic problem and continued medication with over-thecounter topical ointment. The patient did not seek medical assistance for 3 years until a year ago when the bone was visible on the wound base and his family member insisted on treatment.

The medical history revealed long-term sun exposure over a lifetime with no protection from his work as a farmer. Clinical examination revealed a single ulcer on the nose extending to the right lower eyelid and right upper lip, sized $7 \mathrm{~cm} \times 6 \mathrm{~cm}$, base of bone, rolled edge, well-defined border, no undermining, no exudation, no necrosis, and hemorrhage (Figure 1). The heart and lung functions were normal with no comorbidities like hypertension and diabetes mellitus. Laboratory studies including complete blood count, blood biochemistry and urinalysis were within normal limits.
The surgery was done in two stages with the collaboration of oncology surgery and plastic surgery departments. The first stage was tumor removal and LFF reconstruction. The tumor was excised by the oncology surgeon with $2 \mathrm{~cm}$ tumor-free margin, leaving a $12 \mathrm{~cm} x$ $10 \mathrm{~cm}$ defect (Figure 2A). The design of LFF was made along the hairline and inferiorly above the left eyebrow by relying on the dextral temporal artery as pedicle. The defect was closed with no tension. Preservation of nostrils was done by inserting two cut nasogastric tubes (NGT) no. 16 and using 3-0 polypropylene suture for fixation. The flap was sutured using 5-0 polypropylene and 4-0 polyglactin. For intraoral region, the suture was made interlocking with the NGT on the upper side of gingivobuccal mucosa. The raw surface of the forehead was covered with split-thickness skin graft from right thigh (Figure 2B).

Four months later, the second stage surgery was done to create a connection between nostrils and the outer environment. Nasal passage and concha were assessed. Then, moderate-sized holes were made using two NGTs no. 20. The upper lip was sutured to frenulum. Z-plasty on medial aspect of the left eye was done for wider eyeopening. The defect was closed with interrupted sutures using 5-0 polypropylene and 4-0 polyglactin (Figure 3). Six months after the second surgery, there was no sign of recurrence and the patient was planned to continue nasal reconstruction for cosmetic improvement (Figure 4).

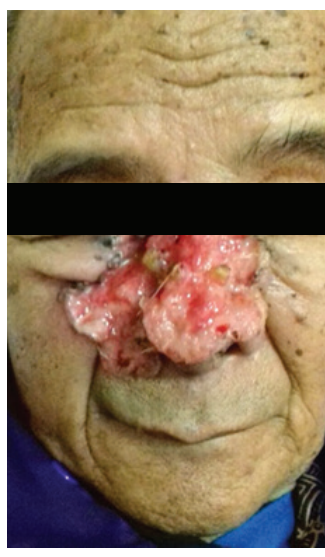

Figure 1. First patient encounter

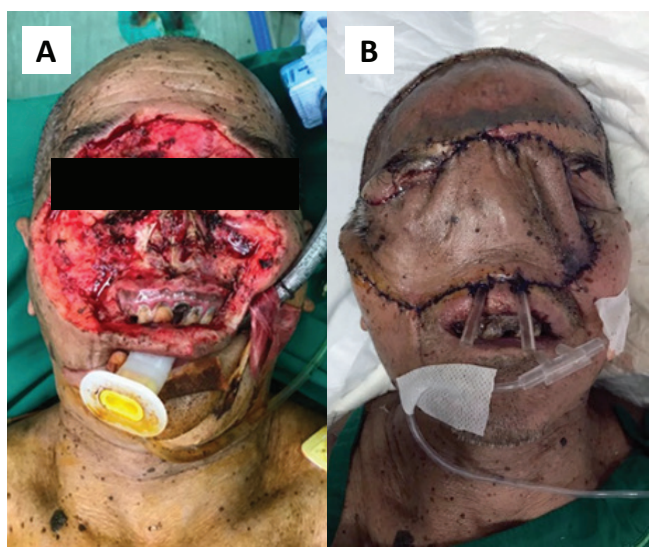

Figure 2. (A) Defect after wide excision; (B) Defect closure with LFF method

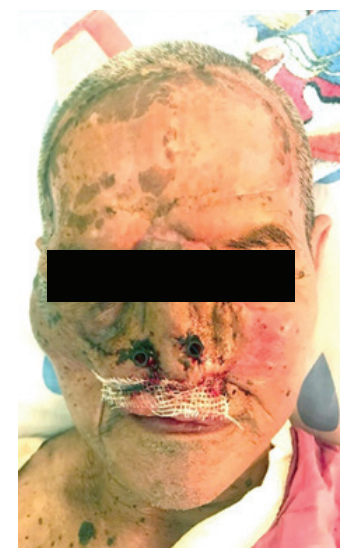

Figure 3. Second surgery 4 months later

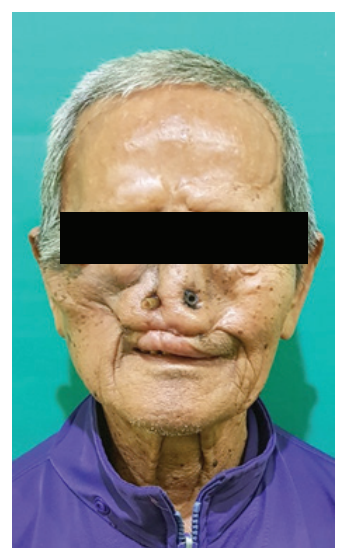

Figure 4. Six months after the second surgery

\section{DISCUSSION}

Neglected BCC cases can still be found in modern days, both in developed and developing countries. Numerous studies on neglected BCC cases pointed to different reasons from the patient and the disease itself. Patient factors include low social milieu, unhygienic culture, low knowledge on skin tumor, old age and multiple medical comorbidities. Disease factors include the painless, slow-growing and low metastatic rate of neoplasm [6,9-10]. In this case, neglect is caused by the economic factor. Interestingly, national health insurance covering the chemotherapy or surgery cost was available, but the patient did not apply due to complicated requirements for application. This means the economic burden was solvable, but the reluctance of the patient due to painless and non-disturbing tumors was the main reason behind the neglect. He was aware of the significant change on his face but chose only to cover it if his daily activities were not impaired. A similar external factor is also found in this case, where 
encouragement from another person urges the patient to seek medical advice.

Neglected BCC contributes to one-third of all giant BCC cases [6]. Unlike common BCC with numerous treatment options from topical to photodynamic therapy or radiotherapy, giant BCC is limited to surgery as the cornerstone of treatment. Nasal GBCC can be especially challenging due to its location and tumor size with a high recurrence rate. Resection of nasal GBCC results in a large complex of soft tissues and bone defects that also require complex reconstructive methods [11]. In this case, wide excision with $2 \mathrm{~cm}$ tumor-free margin was chosen to ensure complete resection of the tumor, leaving the patient with an extensive defect of $>10 \mathrm{~cm}$ in diameter. This is important due to the higher recurrence rate in neglected BCC and giant BCC cases [12].

Reconstructive options should focus on achieving primary wound healing, protecting vital structures, restoring contour, and maximizing functional and aesthetic outcomes [13]. Paramedian forehead flap has become the gold standard for reconstruction of nasal BCC. However, it is not applicable for huge nasal and midface defects in nasal giant BCC, leaving with options such as other local flaps, regional flap, and microsurgical free flap. Considerations in head and neck reconstruction recommend the use of a reverse reconstructive ladder, where the microsurgical free flap becomes the first choice for large or composite defects [14]. The free flap provides new tissues with robust blood supply, enough volume to restore function and form, and resistant to irradiation. However, it has poor skin color match, donor site morbidity, and excessive tissue bulk and requires longer surgery time and special training. Regional flap requires shorter surgery time with easier technique and equipment but still possess the other drawbacks like free flap.

LFF is a local myocutaneous flap supplied by the superficial temporal artery. It was first introduced by McGregor in 1963 for intraoral defect [7] and then modified by Millard and Gillies in 1964 for other facial defects [8]. A single superficial temporal artery can supply the entire forehead skin and muscle due to rich anastomotic plexus linked by choke vessels to forehead angiosomes. This robust vascularization allows for flap elevation without delay or prefabrication and risk of flap necrosis [15]. Nevertheless, the success of LFF also relies on careful preoperative planning including assessment of the defect, hairline height and forehead laxity [16]. LFF has the best skin color match, less tissue bulk, and provides shortest surgery time with the easiest technique or equipment compared to others. A few disadvantages include obvious donor site location, loss of forehead sensation and eyebrow-raise expression [15].

In this case, the preoperative assessment supported LFF as the first option. The forehead area was free of tumor and excision defects, thus available as donor sites. The hairline height was high, $8 \mathrm{~cm}$ with acceptable skin laxity of the forehead to enable tension-free closure. Shorter surgery time with simpler technique of LFF was essential for this old-aged patient to minimize intraoperative complications. The goal of the first surgery was restoring integrity by covering the defect with LFF and maintaining function by putting NGT tubes for breathing on the flap border. The tubes were intentionally not punctured through the flap to avoid flap damage, thus maximizing viability and tissue healing. When the flap had shown good response four months later, another surgery for better function and form was executed for wider left eye-opening and proper position of nostrils. The patient's right eye was not functional before the surgery, thus covering of right eye with flap was not a problem. Six months after the second surgery, the function has been restored as the patient can breathe fine using the tubes. He was also satisfied with cosmetic and unashamed of showing his face. Another nasal reconstruction surgery was planned to further improve cosmetic for a better nose appearance although this might not be necessary due to his old age.

Despite the patient's satisfaction, there are several concerns regarding the use of LFF, especially in the cosmetic aspect. These concerns include loss of facial contours, presence of hair on midface and visible incision line on the forehead. The incision line is of minor concern since it is well hidden near the hairline. The presence of hair can be dealt temporarily by constant shaving or permanently by hair removal laser therapy although the latter option is still rarely executed on flaps. Loss of facial contours, however, is a major concern that requires additional revision surgery. In comparison with distant or free flaps, LFF provides a good functional outcome and lower compromise risk from simpler and faster operation with limitation in the cosmetic outcome from obvious donor site on forehead.

\section{CONCLUSIONS}

Neglected BCC cases still happen mostly due to its slow-growing and painless nature, supported by patient factors such as socioeconomic and educational status. Giant BCCs caused by neglect possess a higher recurrence rate and require wide excision to ensure tumor-free margin, thus leaving a large complex of soft tissues or bone defects to be reconstructed. Lateral forehead flap, being introduced five decades ago, still proves to be a simple yet reliable reconstruction method for extensive nasal defects in nasal GBCCs. This shows that a reconstructive technique from the past is still comparable with various more modern and complicated techniques in selected cases. 


\section{DECLARATIONS}

\section{Competing of Interest}

The authors declare no competing interest in this study.

\section{Acknowledgement}

There are no other people, grants or funds that need to be acknowledged in this study.

\section{REFERENCES}

1. Apalla Z, Lallas A, Sotiriou E, Lazaridou E, loannides D. Epidemiological trends in skin cancer. Dermatol Pract Concept. 2017;7(2):1.

2. American Cancer Society (2018) Facts \& Figures 2018. American Cancer Society, Atlanta. https://www. cancer.org/cancer/basal-and-squamous-cell-skincancer/about/key-statistics.html\#references. Accessed 21 November 2018.

3. Asgari MM, Moffet HH, Ray GT, Quesenberry CP. Trends in basal cell carcinoma incidence and identification of high-risk subgroups, 1998-2012. JAMA Dermatology. 2015;151(9):976. https://doi. org/10.1001/jamadermatol.2015.1188.

4. Malec K, Brzewski P, Cenda P, Kuchta K. Gasinski M, Wojas-Pelc $A$, et al. Extensive, neglected basal cell carcinoma of the half of the face-surgical treatment and reconstruction with an anterolateral microvascular thigh flap. Adv Dermatol Allergol. 2016;33(3):235-8.

5. Larsen AK, Ghulam W, El-Charnoubi. Gehl J, Krag C. Neglected giant scalp basal cell carcinoma. Plast Reconstr Surg Glob Open. 2014;2:e120.

6. Varga E, Korom I, Rasko Z, Kis E, Varga J, Olah J, et al. Neglected basal cell carcinoma in the 21st century. J Skin Cancer. 2011;2011:392151.
7. McGregor IA. The temporal flap in intra-oral cancer: its use in repairing the post-excisional defect. $\mathrm{Br} \mathrm{J}$ Plast Surg. 1963;16:318-35.

8. Millard DR, Jr. Forehead flap in immediate repair of head, face and jaw. Am J Surg. 1964;108:508-13.

9. Bisgaard E, Tarakji M, Lau F, Riker A. Neglected skin cancer in the elderly: a case of basosquamous cell carcinoma of the right shoulder. J Surg Case Rep. 2016;2016(8):rjw134.

10. Wandrey N, Chen T, Eng T. Dramatic resolution of an unresectable giant basal cell carcinoma treated with intensity-modulated radiation therapy (IMRT) - a case report. Cureus. 2015;7(12):e416.

11. Lackey PL, Sargent LA, Wong L, Brzezienski M, Kennedy JW. Giant basal cell carcinoma surgical management and reconstructive challenges. Ann Plast Surg. 2007;58(3):250-4.

12. Archontaki M, Stavrianos SD, Korkolis DP, Arnogiannaki $\mathrm{N}$, Vassiliadis V, Liapakis IE, et al. Giant basal cell carcinoma: clinicopathological analysis of 51 cases and review of the literature. Anticancer Res. 2009;29(7):2655-63.

13. Arslan H, Güzel MZ, Cnar C. Treatment of giant basal cell carcinomas of the head and neck with aggressive resection and complex reconstruction. J Craniofac Surg. 2012;23(6):1634-7.

14. Chim H, Salgado CJ, Seselgyte R, Wei FC, Mardini S. Principles of head and neck reconstruction: an algorithm to guide flap selection. Semin Plast Surg. 2010;24(2):148-54.

15. Supit L, Sudjatmiko G. The extended lateral forehead flap: today as was 50-years ago. Jurnal Plastik Rekonstruksi. 2012;1(4):374-80.

16. Boyd CM, Baker SR, Fader DJ, Wang TS, Johnson TM. The forehead flap for nasal reconstruction. Arch Dermatol. 2000;136:1365-70. 